\title{
FAULT DETECTION WITHIN A COMPONENT OF A SYSTEM OF COMMUNICATING FSMS
}

\author{
Ana Cavalli ${ }^{1}$, Svetlana Prokopenko ${ }^{1}$, Nina Yevtushenko ${ }^{2}$ \\ 1 Institut National des Telecommunications, 9 rue Charles Fourier 91011 Evry France \\ Email: Ana.Cavalli@int-evry.fr, Svetlana.Prokopenko@int-evry.fr \\ 2 Tomsk State University, 36 Lenin av. 634050 Tomsk Russia \\ Email:Yevtushenko@elefot.tsu.ru
}

\begin{abstract}
In this paper, we consider the problem of detecting faults within a component of a system of communicating FSMs. These faults are detected by a given set of external test cases, which satisfy appropriate test purposes. Given a component FSM and an external test case, we propose a procedure for deriving the set of all output faults of the component machine, which can be detected with this test case. Applications range from test suite minimization to derivation of diagnostic tests for a component FSM. In this paper, we use the above technique for augmenting a given test suite in order to detect all output faults within a component under test. The same approach can be used with respect to any other finite set of faults within the implementation of a component FSM.
\end{abstract}

Key words: Finite State Machine (FSM), communicating FSMs, test suit

\section{INTRODUCTION}

There are a number of methods for test suite derivation based on an FSM model. Some methods return a test suite, which satisfies appropriate test purposes [1,2]; other return a complete test suite w.r.t. an appropriate fault domain [3-10]. Traditional test purpose approaches are based on the following idea: to traverse an appropriate set of transitions. If each transition is traversed then the obtained test suite is known to detect any output fault of an implementation system and to have a rather good quality. However, usually traversing all the transitions is impossible for a large system and a 
test engineer selects a subset of them. There are a number of papers [see, for example, 1, 2] with recommendations how to select such a subset in the best way. When coming to communicating FSMs the test purpose often is to traverse each possible transition of each component FSM [2, 11]. In the case of communicating FSMs, each transition of the overall system comes from a combination of transitions of some modules. By this reason, a proper subset of all the global transitions that usually is much less than the set of all global transitions satisfies such a test purpose. The fault coverage of the obtained test suite remains unknown. In the paper [10], the authors show that under the assumption that we can observe internal outputs of each component FSM a test suite traversing each possible transition of each component FSM detects any single output fault of each component FSM.

In this paper, we continue to study a fault coverage of the above test suite. A method is proposed for determining which output faults of an implementation component FSM are detected with a given test suite when no access is granted to the internal outputs of an appropriate component machine. We also show how these faults influence a behavior of the overall implementation. In particular, we show how to relate to each test case of a given external test suite a finite set of output faults of a possible implementation of a component under test that can be detected with this test case and how the approach can be used for test minimization without a loss of completeness.

The novelty of the work presented in this paper is that we consider all output faults of the component FSM, not only single output faults. We illustrate that when no access to internal outputs is granted it is not enough to traverse an appropriate transition of the component even for detecting a single output fault. Additional test cases are necessary to guarantee complete fault coverage and we propose a technique how to derive them.

\section{PRELIMINARIES}

\subsection{I/O Finite State Machines}

An I/O finite state machine (often simply called a FSM or a machine throughout this paper) is an initialized, possibly partially specified, deterministic machine denoted by a 6-tuple $A=\left(S, X, Y, \delta, \lambda, D_{A}, s_{0}\right)$ where $S$ is a finite set of states with $s_{0} \in S$ as the initial state, $X$ is a finite nonempty set of inputs, $Y$ is a finite nonempty set of outputs, while $\delta$ and $\lambda$ are next state function $\delta: D_{A} \rightarrow S$ and output function $\lambda: D_{A} \rightarrow Y$, where $D_{A} \subseteq S \times X$ is the specification domain. The machine $A$ is complete if $D_{A}=S \times X$; otherwise, the machine is partial. In the usual way, the function $\lambda$ is extended to input 
sequences with results in the set of output sequences. The set $I_{A}$ is the set of all input sequences where a behavior of the FSM $A$ is defined. The $\lambda(\alpha)$ denotes the output sequence that is produced by the FSM when an acceptable sequence $\alpha$ of the set $I_{A}$ is submitted. As usual, given input sequence $x_{1} \ldots x_{k}$ and output sequence $y_{1} \ldots y_{k}$, the sequence $x_{1} / y_{1}, \ldots, x_{k} / y_{k}$ is called $a$ trace of the $A$ if $\lambda\left(x_{1} \ldots x_{k}\right)=y_{1} \ldots y_{k}$.

Given two FSMs $A=\left(S, X, Y, \delta, \lambda, D_{A}, s_{0}\right)$ and $B=\left(T, X, Y, \Delta, \Lambda, D_{B}, t_{0}\right)$, FSM $A$ is said to be a reduction of FSM $B$, denoted $A \leq B$, if the set of traces of $A$ is a subset of that of the FSM $B$. For partial deterministic FSMs the reduction relation coincides with a so-called quasi-equivalence relation [12]. Deterministic FSM $A$ is a reduction of deterministic FSM $B$ if and only if $B$ is quasi-equivalent to $A$.

\subsection{Composition of FSMs}

We consider a system composed by several FSMs $C_{i}, i=1, \ldots, k$, as shown in Figure 1. We refer to symbols of alphabets $X_{i}$ as to external inputs, to symbols of alphabets $Y_{i}$ as to external outputs while referring to symbols of alphabets $Z_{i j}, i \neq j$, as to internal actions. In fact, some alphabets can be empty but the union of $X_{i}\left(Y_{i}\right)$ over all $i \in I$ is not empty. We refer to the component FSM as to an embedded FSM if the component FSM has no external inputs and outputs. The system always has a single message in transit, i.e. the alphabets are pairwise disjoint and the environment submits the next input only when the system has produced an output to the previous input. Under this assumption, a component machine accepting an input may produce either an external or a single internal output. If the component machines fall into infinite internal dialog when an appropriate external input sequence is submitted we say the system falls into live-lock under the input sequence and its behavior is said to be not specified. If the behavior of one of component FSMs is not specified under a submitted input we also assume the system behavior is not specified. Under the above assumptions we can simulate a behavior of the composition under an external input sequence. 


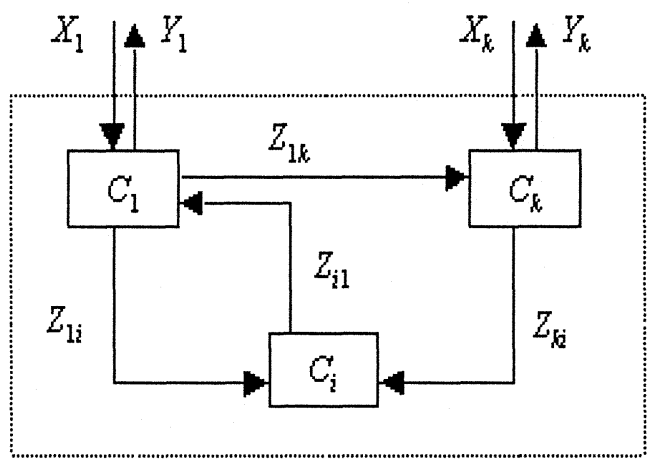

Figure 1. System of communicating FSMs.

Let a component FSM $C_{i}$ have the set $Q_{i}$ of states. Then the composite FSM is a FSM over the union of alphabets $X_{i}$ and $Y_{i}$ with the state space that is a subset of the Cartesian product of $Q_{i}, i \in I$. States of the Cartesian product are divided into stable and transient states. By definition, the initial state $q_{10}$ $\ldots q_{k 0}$ is stable. Otherwise, the state is stable if it has been reached immediately after the system has produced an external output. We start from the initial state $q_{10} \ldots q_{k 0}$. The algorithm for simulating a behavior of the system w.r.t. an external input sequence is based on the derivation of a partial reachability graph induced by the input sequence $[5,10]$ that then is projected onto external alphabets. The composite reference FSM $R M=\diamond C_{i}$, $i=1, \ldots, k$, can be derived using the above procedure. In this case, we derive the global reachability graph [4].

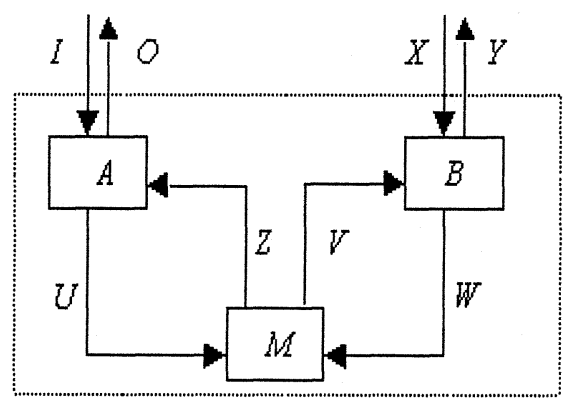

Figure 2. System under test. 


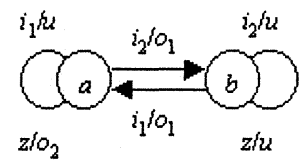

$a$

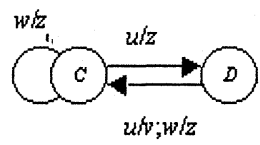

b

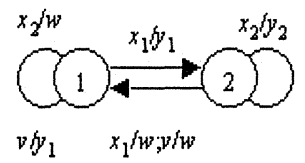

$c$

Figure 3. Component FSMs $A(a), M(b)$ and $B(c)$.

We illustrate the procedure by a simple working example that has communicating FSMs $A, B$ and media $M$ through which component machines $A$ and $B$ communicate (Figures 2 and 3). Media $M$ has no external inputs and outputs and can be considered as an embedded component FSM. Consider external input sequence $x_{1} x_{1}$. When $x_{1}$ is applied at the initial state $a C 1$ of the system the component $B$ produces external output $y_{1}$ and enters state 2 . Thus, the system produces the output $y_{1}$ and enters a stable state $a C 2$ when the input $x_{1}$ is submitted. When the next input $x_{1}$ is applied to the reference system at stable state $a C 2$ the component $B$ produces internal output $w$ and came back to state 1, i.e. the system enters a transient state $a C 1$. The media $M$ at state $C$ replies with output $z$ to input $w$ and remains at state $C$, i.e. the system remains at the transient state $a C 1$. Input $z$ is then applied to $A$ at state $a$; the $A$ produces external output $o_{2}$, i.e. the system reaches the stable state $a C 1$. The composite FSM is shown in Figure 4. However, we do not need to construct the composite FSM; the reference external output response to an external input sequence is derived by simulating a system behavior under the input sequence.

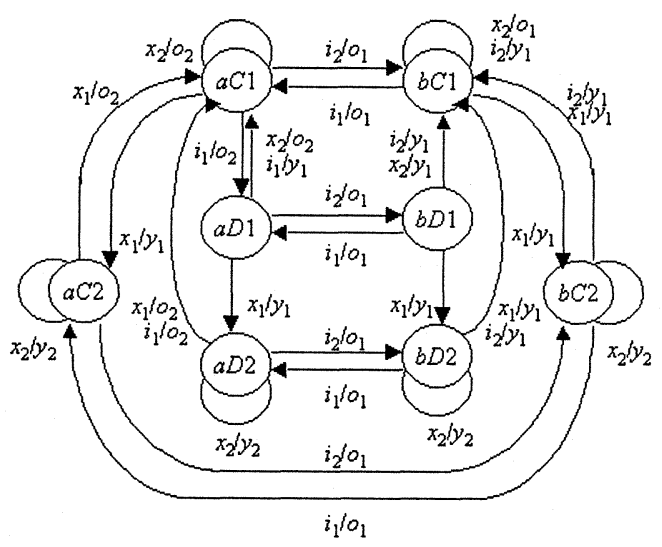

Figure 4. The composite FSM. 


\subsection{Fault domain}

One important aspect of test generation is to specify an appropriate fault model. As mentioned above, it is natural to assume that the structure of the reference system is preserved in all potential implementations and at most one component FSM can be faulty [see, for example, 5, 13, 14, 15]. We refer to a FSM modeling the reference system as to the reference FSM while calling the fault domain the set $\mathfrak{R}$ of FSMs modeling all possible systems under test. As usual, we assume an implementation is completely specified, i.e. any input sequence can be applied to the implementation. Moreover, each component FSM can produce an error signal to the environment if an applied input is unexpected for the specification component FSM at a current state. The implementation FSM $I M \in \mathscr{R}$ is called a faulty or a nonconforming implementation if the reference FSM is not a reduction of $I M$; otherwise it is a conforming implementation.

A test suite is a finite set of finite input sequences of the reference machine $R M$. A test suite, which detects any nonconforming implementation, is called a complete test suite w.r.t. the fault domain $\mathscr{R}$. Thus, if a system under test is modeled by an FSM $I M \in \mathfrak{R}$ and is not detected by a complete test suite w.r.t. $\Re$ then one concludes that the reference system is a reduction of the system under test, i.e. a behavior of the system under test coincides with that of the reference system under any input sequence where the behavior of the reference system is specified.

In this paper, we are concerned about detecting output faults in implementations of component FSMs. A test suite is complete if it detects all output faults in the implementation of each component FSM. As a guide, we use a widely used test purpose for a system of communicating FSMs [16]. A test suite satisfying the test purpose must traverse each possible transition of each component FSM in the reference system. Test selection is performed in order to obtain a test suite satisfying the above test purpose such that the result test suite detects the maximum of output faults within implementations of component FSMs.

Our first step is to evaluate which output faults in the implementation of a component FSM can be detected by a test suite traversing each transition of the component that is involved in interaction with other component FSMs. To describe these faults we use an approach originally proposed in [8] for test minimization for an embedded component and developed in [10] for single output faults. 


\section{DETECTING OUTPUT FAULTS IN A COMPONENT MACHINE WITH AN EXTERNAL TEST CASE}

We consider a reference system composed by $k$ deterministic FSMs, as shown in Figure 1. Let $\alpha$ be an external test sequence, i.e. a sequence over external input alphabet. If we are concerned only about faults within a component FSM $C_{i}$ we need to describe which output faults of the component FSM can be detected with the sequence $\alpha$. We further assume that live-locks in the implementation system can be detected by a timer and show below that all the faults of the component FSM $C_{i}$ detected by $\alpha$ can be described by a collection of finite subsets of output faults.

\subsection{Detectable output faults}

Our first step is to illustrate a relation between an external test case and output faults in a component machine, which can be detected with the test case. Consider the composition of FSMs $A, B$ and $M$ shown in Figure 2 and input sequence $x_{1} x_{2}$. Suppose that implementations of machines $A$ and $M$ are faulty-free while an implementation $\operatorname{Imp}_{B}$ of FSM $B$ can have output faults. The reference composition has an external output response $y_{1} y_{2}$ to $x_{1} x_{2}$. We are interested which faults in the implementation $\operatorname{Imp}_{B}$ of the component FSM $B$ can be detected with the external test case $x_{1} x_{2}$.

Let $x_{1}$ be applied when component FSMs $A, M$ and $B$ are at their initial states $a, C$ and 1. The implementation FSM $\operatorname{Imp} p_{B}$ produces $y_{1}, y_{2}$ or $w$ to the input $x_{1}$ and enters state 2 . If the implementation machine $\operatorname{Imp} p_{B}$ at the initial state produces the output $y_{2}$ to $x_{1}$ then the overall implementation system produces an unexpected external output response $y_{2}$ to the test case $x_{1}$. Therefore, if the $\operatorname{Imp}_{B}$ has the output fault $\left(1, x_{1}, y_{2}\right)$ then the implementation system is detected with the test case $x_{1}$ independently of its other output faults. Let the implementation machine $\operatorname{Imp}_{B}$ at the initial state produce the output $w$ to $x_{1}$ that then is applied to the machine $M$ at the initial state $C$. The machine $M$ produces the output $z$ to $w$ and remains at the state $C$. The output $z$ is applied to the machine $A$ at the state $a$ and $A$ produces an unexpected output response $o_{2}$ to $x_{1}$. Thus, if the $\operatorname{Imp}_{B}$ has the output fault $\left(1, x_{1}, w\right)$ then an implementation system is detected with the test case $x_{1}$ independently of other output faults.

Let the implementation machine $\operatorname{Imp}_{B}$ at the initial state produce the reference output $y_{1}$ to $x_{1}$ and enter state 2 . We then apply the next input $x_{2}$ to the implementation system. The implementation machine $\operatorname{Im} p_{B}$ at the state 2 produces $y_{1}, y_{2}$ or $w$ to $x_{2}$. If the $\operatorname{Im} p_{B}$ produces $y_{1}$ the overall system produces 
unexpected output $y_{1}$ to $x_{2}$. Thus, if the $\operatorname{Imp}_{B}$ has output fault $\left(2, x_{2}, y_{1}\right)$ it is detected with the test case $x_{1} x_{2}$. If the $\operatorname{Imp}_{B}$ at the state 2 produces the output $w$ to $x_{2}$, it remains at the state 2, and then after some transitions where $\operatorname{Imp} p_{B}$ is not involved the implementation system produces $o_{2}$ to $x_{2}$. Thus, when the $\operatorname{Imp} p_{B}$ has the output fault $\left(2, x_{2}, w\right)$ it is detectable with the test case $x_{1} x_{2}$. The construction is illustrated in Figure 5 where wrong external outputs are shown in bold.

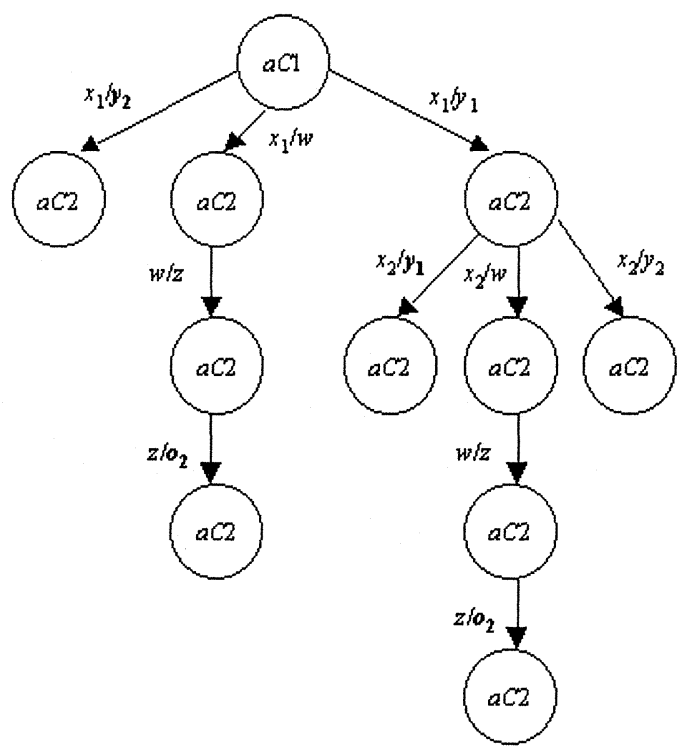

Figure 5. An example of the detection of output faults of the component FSM with an external test case.

The above example clearly shows that given an external test case and a component under test, we can construct the set of all output faults detected within the component machine with the given test case. However, the number of these sets may be exponential. We obtain most interesting case when an external test case completely tests an appropriate transition. These faults are detected by a test case independently of other faults within the implementation of the component FSM. In fact, such a test case detects $|Y|^{|\mathcal{Q} \times X|-1}$ output faults where $Q$ is the state set while $X$ and $Y$ being the component's input and output alphabets. Here we notice that to completely check a transition usually it is not enough to traverse this transition.

Given an external input sequence $\alpha$ and a set Out $_{i}$ of output faults of the component FSM $C_{i}$, the set Out $_{i}$ is detectable with $\alpha$ if any implementation system where the set of output faults of an implementation of the $C_{i}$ contains $\mathrm{Out}_{i}$, has an unexpected output response to $\alpha$ at Points of Observation (POs). 
If a test case completely checks a component transition then for short, we include the only transition into the set $\mathrm{Out}_{i}$.

\subsection{Constructing the set of detectable output faults}

Given a component FSM $C_{i}$ that can be faulty, and an external input sequence $x_{1} \ldots x_{m}$, we first derive an acceptor $F\left(C_{i}, x_{1} \ldots x_{m}\right)$ that represents all possible composition traces when an implementation of each component machine different from $C_{i}$ is faulty-free. By a designated state Fail the acceptor recognizes those traces that imply an unexpected behavior of the composition. The acceptor is derived based on the component FSMs and is then projected onto Input/Output $(\mathrm{I} / \mathrm{O})$ pairs of the component $C_{i}$ in usual way [10]. I/O pairs of the components different from $C_{i}$ are replaced with the empty word. To determinize the obtained acceptor we use a subset construction and replace each subset having the state Fail by a designated state Fail without outgoing transitions. Moreover, each state, for which there exists an input that takes the projection only to the Fail state, is replaced with the Fail state. The expected external output response $y_{1} \ldots y_{m}$ to input sequence $x_{1} \ldots x_{m}$ is obtained by simulating a behavior of the reference system applying the input sequence.

Formally, the state set of the acceptor $F\left(C_{i}, x_{1} \ldots x_{m}\right)$ is a subset $P$ of $Q_{1} \times \ldots \times Q_{i-1} \times Q_{i} \times Q_{i+1} \times \ldots \times Q_{k} \times\{0, \ldots, m\}$. The acceptor is constructed for inputs $x_{1}, \ldots, x_{m}$ in succession. States of the set $P$ are divided into stable and transient states. By definition, the initial state $q_{10} \ldots q_{k 0} 0$ is stable. Otherwise, the state is stable if it has an incoming transition with an external output. The stable states cannot be merged with transient states. Two transient states with the same names in the segment for the same external input are merged if they have an incoming transition labeled with a pair with the same output part. We start from the initial state $\left(q_{10} \ldots q_{k 0}\right) 0$.

Given a state $\left(q_{1 t} \ldots q_{k t}\right) j$ and I/O pair $a / b$ of the component $C_{p}$, the $a / b$ takes the acceptor from state $\left(q_{1 t} \ldots q_{p t} \ldots q_{k t}\right) j$ to a stable state $\left(q_{1 t} \ldots q_{p t}^{\prime} \ldots\right.$ $\left.q_{k t}\right)(j+1)$ if $b$ is the expected external output of the system to input $x_{j+1}$. Here $q_{p j}^{\prime}$ is the state where the input $a$ takes the component FSM $C_{p}$ from the state $q_{p i}$. If $b$ is an unexpected external output of the system to input $x_{j+1}$ or $a$ is an unexpected input at state $q_{p i}$ then the $a / b$ takes the acceptor from state $\left(q_{1 t} \ldots\right.$ $\left.q_{k t}\right) j$ to the designated state Fail. If $b$ is an internal output of the component machine $C_{j}$ then the $a / b$ takes the acceptor from state $\left(q_{1 t} \ldots q_{j t} \ldots q_{k t}\right) j$ to a transient state $\left(q_{1 t} \ldots q_{j t}^{\prime} \ldots q_{k t}\right) j$. The $a / b$ also takes the acceptor to the state Fail if there appears a cycle labeled only with internal actions in the acceptor. The only difference between the component FSM $C_{i}$ of our interest and other component FSMs is that we consider all possible outputs to the input $a$ when $a$ is applied to the $C_{j}$ which keep the deterministic 
implementation of $C_{i}$, while considering only the reference output for all other component FSMs. We denote $D\left(C_{i}, x_{1} \ldots x_{m}\right)$ the deterministic projection of $F\left(C_{i}, x_{1} \ldots x_{m}\right)$ onto I/O pairs of the component FSM $C_{i}$. To reduce the projection we replace with the Fail state each state for which there exists an input that takes the projection only to the Fail state.

By construction, the acceptor $D\left(C_{i}, x_{1} \ldots x_{m}\right)$ is an acyclic graph and comprises complete information about all sets of detectable output faults of the component FSM of our interest.

We say a state $p$ of the acceptor $D\left(C_{i}, x_{1} \ldots x_{m}\right)$ different from the Fail state is deterministically reachable from the initial state if for each prefix $\alpha$ of the input sequence taking the acceptor from the initial state to state $p$ there is the only output sequence $\beta$ such that $\alpha / \beta$ does not take the acceptor from the initial state to the Fail state.

For each state $p$ deterministically reachable from the initial state in the acceptor $D\left(C_{i}, x_{1} \ldots x_{m}\right)$ and every branch from the state $p$ to the Fail state, we derive the set Out $_{i}$ including into the set all transitions of the branch. To reduce the obtained collection we remove each item that comprises another item and denote $\operatorname{Out}\left(C_{i}, x_{1} \ldots x_{m}\right)$ the obtained set system.

Theorem 3.1. Given an external input sequence $\alpha$, a nonconforming implementation $I m p_{i}$ of $C_{i}$ is detectable with $\alpha$ if and only if it has a set of output faults that contains an item of the set system $\operatorname{Out}\left(C_{i}, \alpha\right)$.

In fact, an implementation system with component FSMs $C_{1}, \ldots, C_{i-1}$, $\operatorname{Imp}_{i}, C_{i+1}, \ldots, C_{k}$ is detected by $\alpha$ if and only if it has a trace that takes the acceptor $F\left(C_{i}, \alpha\right)$ from the initial state to the designated state Fail. The latter is possible if and only if the component machine $\operatorname{Imp}_{i}$ has the set of output faults that contains I/O pairs of the component FSM which occur through the corresponding projection of the path, i.e. has the set of output faults that contains an item of the set system $\operatorname{Out}\left(C_{i}, \alpha\right)$.

The set system $\operatorname{Out}\left(C_{i}, \alpha\right)$ can be reduced if some test cases are already included in the test suite. In this case, constructing the acceptor we do not have to consider at least all wrong I/O pairs of the component $C_{i}$ of the transitions completely tested with other test cases. We denote $\operatorname{Out}\left(C_{i}, \alpha / T\right)$ such a system where $T$ is the part of a test suite that is already derived. For example, in our working example, $\operatorname{Out}\left(A, i_{2} i_{2} i_{2} /\left\{i_{2} x_{1} x_{1} i_{2}\right\}\right)$ is empty.

\section{APPLICATION TO TESTING COMMUNICATING FSMS}

Theorem 3.1 provides a guide of what part of the behavior of a component FSM can be tested with a given test suite. The above result can be used in various applications, which range from test selection, test suite 
minimization to derivation of diagnostic tests for a component FSM. Test minimization w.r.t. arbitrary faults in the implementation of a component FSM has been considered in $[8,17]$. In this paper, we consider how the above construction can be used for test minimization w.r.t. output faults and for selecting test cases.

\subsection{Test selection for a system of communicating FSMs based on a composite FSM}

When a test suite is derived for a system of communicating FSMs the test purpose usually is to traverse every possible transition of each specification component FSM at least once. Test selection is performed based on the test purpose. We here propose how to reach the test purpose while having a test suite with a higher fault coverage. The technique is based on the composite FSM; however, it can easily be modified in order to use only parts of the composite machine with a predefined length of input sequence.

In the paper [16] the method of test derivation based on the composite FSM is presented. The idea of the method is as follows.

Every transition of a component FSM is involved in a number of global transitions. We assign a distinguished color for every transition of each component FSM and use this color for each global transition where the component transition is involved. To reach the test purpose we need to find a minimum set of input sequences of the composite FSM, which cover all the colors.

Using the results of the previous section we can associate with each test case the set of output faults within a component under test which are detected with the test sequence. For example, given an input sequence, we can consider the number of completely tested transitions as scope of the sequence. In this case, we are required to derive a minimum set of input sequences with maximum scope, which cover all the colors. Using the above technique we will reach the test purpose and simultaneously the obtained test suite will have the good fault coverage, in particular, w.r.t. output faults in implementations of component FSMs.

Consider the composition of three FSMs $\operatorname{Imp}_{A}, M$ and $B$ and a test case $i_{2}$. By direct inspection (Figure 6), one can assure the test case traversing the transition $\left(a, i_{2}\right)$ within the component FSM $A$ does not completely check this transition. 


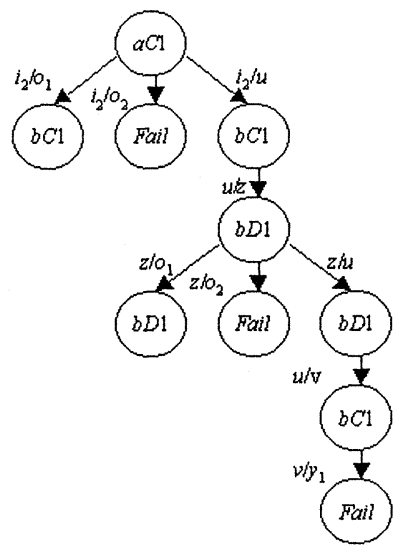

Figure 6 . The test case traversing the transition $\left(a, i_{2}\right)$.

If the implementation $I m p_{A}$ of the component FSM $A$ has two output faults $\left(a, i_{2}, u\right)$ and $\left(b, z, o_{1}\right)$, i.e. $I m p_{A}$ is a submachine of the FSM shown in Figure 7, then the composition of $I m p_{A}, B$ and $M$ has the reference output $o_{1}$ to $i_{2}$. However, this composition reaches the state $b D 1$ that is different from the state $b C 1$ of the reference composition. Therefore, to detect this fault we can combine a non-deterministic FSM presented in Figure 7 with component FSMs $B$ and $M$ and to derive an input sequence distinguishing reference state $b C 1$ from the state $b D 1$ of the obtained composition.

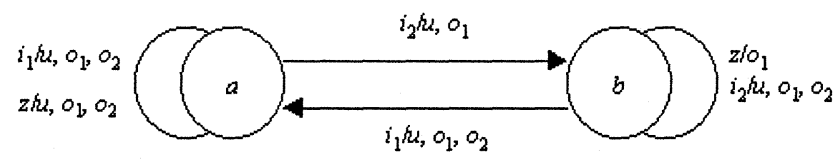

Figure 7. The implementation $\operatorname{Imp} p_{A}$.

As it is shown in Figure 8, in our case, such a sequence exists and is equal to $i_{2} i_{2}$. The reference system at the state $b C 1$ has the output $y_{1} y_{1}$ to $i_{2} i_{2}$ while for the composition where the output fault $\left(a, i_{2}, u\right)$ occurs, we have at the state $b D 1$ outputs $o_{1}, o_{2}$ or $y_{1}$ to $i_{2}$. If the faulty implementation produces the reference output $y_{1}$ to $i_{2}$ then it produces the unexpected output $o_{1}$ to the next $i_{2}$, since at state $b$ the implementation component FSM $\operatorname{Im} p_{A}$ produces $o_{1}$ to $z$ (Figure 7). Therefore, the transition $\left(a, i_{2}\right)$ is completely tested by the test case $i_{2} i_{2} i_{2}$. 


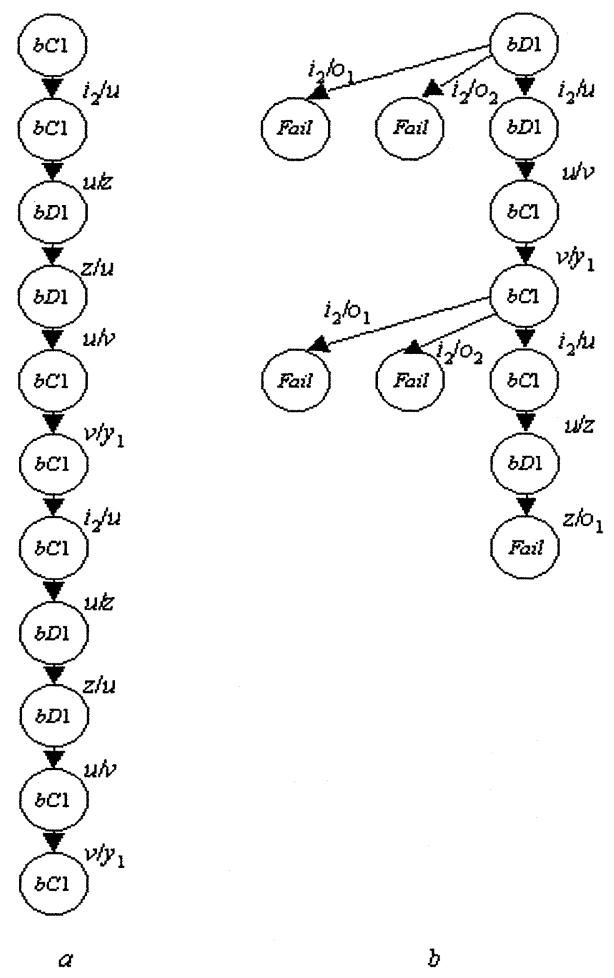

Figure 8. An input sequence distinguishing the reference state $b C 1(a)$ from the state $b D 1(b)$ of the composition where an implementation of component FSM $A$ has output faults $\left(a, i_{2}, u\right)$ and $\left(b, z, o_{1}\right)$.

The method for deriving input sequences distinguishing states of two non-deterministic FSMs is proposed in [18]. However, if the reference state cannot be distinguished from the given state of the obtained composition we do not guarantee that it cannot be distinguished at all. Possibly, the cause is the compact representation of all possible implementations as a unique nondeterministic FSM. In this case, one can try to add to a given test case all input sequences of length up to an appropriate integer, say two, and check if the transition can be completely tested. If no then we give up to completely test the transition and add nothing to the given test case. The latter approach can also be applied in the case when the reference composite FSM is not derived.

The procedure can be shortened when some of test cases are already included into the test suite. In this case, we are not to check transitions, which are completely tested by included test sequences.

For our working example, a test suite completely checking all transitions of the component FSM $A$ is $T S=\left\{x_{2} i_{1} x_{1} x_{1} ; i_{2} i_{1} x_{1} x_{2} i_{2} ; i_{2} x_{1} x_{1} i_{2} i_{2} ; i_{2} x_{1} x_{2} i_{1} ; i_{2} i_{2} i_{2}\right\}$. 
By direct inspection, one can assure the same test suite checks each transition of FSM $M$ and FSM $B$.

\subsection{Test suite minimization w.r.t. output faults of component FSMs}

When a test suite has been derived from the reference composite FSM [see, for example, 19, 20] it can be expected to be redundant when only one component FSM can be faulty. In this section, we propose a procedure for test suite minimization without a loss of its fault coverage w.r.t. output faults of the component FSMs. In particular, the procedure can be used for minimization of interoperability tests [21, 22].

Given a complete test suite w.r.t. an appropriate fault model, we can minimize it without loss of its fault coverage based of Theorem 3.1 under an assumption that at most component FSM $C_{i}$ can be faulty.

Theorem 4.1. Given a complete test suite $T S$ for the reference FSM $R M=\diamond C_{j}$ w.r.t. the fault domain $\mathfrak{I}$ and proper subset $T \subset T S$, let $\mathfrak{R}$ be the set containing each FSM of the fault domain $\mathfrak{I}$ that is a composition of the component FSMs $C_{1}, \ldots, C_{i-1}, \operatorname{Imp}_{i}, C_{i+1}, \ldots, C_{k}$ where $\operatorname{Imp}_{i}$ is an implementation of $C_{i}$, possibly with output faults. If each set of output faults detected by test cases of the test suite $T S$ also is detected with an appropriate test case of the set $T$ then the set $T$ is a complete test suite w.r.t. the fault domain $\mathfrak{R}$.

In other words, if a proper subset $T$ of a given test suite $T S$ satisfies the conditions of Theorem 4.1 then $T$ and $T S$ detect the same set of output faults within the component FSM $C_{i}$. Here we notice that conditions of Theorem 4.1 are sufficient but not necessary. The reason is the sets of detectable output faults may be changed if some sequences are already included into the test suite.

We now propose a straightforward procedure for test minimization for a system of communicating FSMs under an assumption that at most one component FSM can be faulty. Given a test suite $T S$ for a system of communicating FSMs and the sets $\operatorname{Out}_{\alpha}\left(C_{i}\right), \alpha \in T S$, where $\operatorname{Out}_{\alpha}\left(C_{i}\right)$ is a set of output faults of $C_{i}$ detected with $\alpha$, the procedure returns subset $T$ of $T S$ that detects the same set of faulty implementations of each component FSM $C_{i}$ as the initial test suite $T S$. We enumerate test cases in $T S=\left\{\alpha_{1}, \ldots, \alpha_{k}\right\}$ and iteratively for each test case $\alpha_{j}$, consider $T=T S \backslash\left\{\alpha_{j}\right\}$. If $\operatorname{Out}\left(C_{i}, \alpha_{j} / T\right)$ is empty then we can delete $\alpha_{j}$ from the current test suite TS without a loss of its completeness.

Here we notice that in the proposed approach we do not enumerate all the output faults, a number of which is exponential. We try to treat output faults in parallel. However, the result returned by the above procedure usually 
depends on the order of the test cases in the set TS. Just now we can only recommend to try first the test case that completely tests a maximum number of transitions. Another procedure based on a Boolean matrix [8] can be proposed for a test suite minimization. In this case, the problem of determining a minimum subset of a given test with the same fault coverage reduces to the problem of deriving a minimum cost cover of a Boolean matrix.

Given test suite $T S=\left\{x_{2} i_{1} x_{1} x_{1} ; i_{2} i_{1} x_{1} x_{2} i_{2} ; i_{2} x_{1} x_{1} i_{2} i_{2} ; i_{2} x_{1} x_{2} i_{1} ; i_{2} i_{2} i_{2}\right\}$ for our working example, by direct inspection one can assure that its subset $\left\{x_{2} i_{1} x_{1} x_{1} ; i_{2} x_{1} x_{1} i_{2} ; i_{2} x_{1} x_{2} i_{1}\right\}$ detects the same set of output faults in implementations of the component FSMs. Moreover, the test case $i_{2} x_{1} x_{2} i_{1}$ cannot be replaced with $i_{2} i_{1} x_{1} x_{2} i_{2}$ without a loss of its fault coverage, because the latter does not check a transition $\left(a, i_{2}\right)$ completely, despite of the fact that both subsets of the initial test suite traverse the same set of transitions in each component FSM.

\section{CONCLUSION}

In this paper, given a system of communicating FSMs, we establish a relation between an external test case and output faults of a component machine, which can be detected with this test case. We also demonstrate an application of the established relation for test selection and minimization for communicating FSMs. The relation can also be useful when calculating fault coverage of a given external test suite w.r.t. faults within the implementation of a component FSM [23] as well as when deriving diagnostic tests for output and transfer faults of a component FSM.

\section{REFERENCES}

[1] J.-C. Fernandez, C. Jard, T. Jeron, and C. Viho, Using on-the-fly verification techniques for the generation of test suites. In CAV, LINCS 1102, USA, July, 1996.

[2] Cavalli, A., Lee, D., Rinderknecht, C., and Zaidi, F., Hit-or-Jump: An algorithm for embedded testing with applications to IN services. Proceedings of Joint Inter. Conf. FORTE/PSTV99, pp: 41-58.

[3] Petrenko, A., Yevtushenko, N. and v.Bochmann, G. (1996), Testing deterministic implementations from their nondeterministic specifications. Proceedings of 9th IWTCS, pp: 125-140.

[4] Petrenko A., Yevtushenko, N., and v. Bochmann, G. (1996), Fault models for testing in context. Proceedings of Joint Inter. Conf. FORTE/PSTV96.

[5] Lima, L.P., and Cavalli, A.R. (1997), A pragmatic approach to generating test sequences for embedded systems. Proceedings of 10th IWTCS, pp: 288-307. 
[6] Lima, L.P., and Cavalli, A.R. (1997), Application of embedded testing methods to service validation. Second IEEE Intern. Conf. on Formal Engineering methods.

[7] A. Petrenko, N. Yevtushenko, Testing faults in embedded components. Proceedings of $10^{\text {th }}$ IWTCS, pp: 272-287.

[8] Yevtushenko, N., Cavalli, A.R., and Lima, L.P. (1998), Test minimization for testing in context. Proceedings of 11th IWTCS, pp: 127-145.

[9] Lima, L.P. (1998), A pragmatic method to generate test sequences for embedded systems. Ph.D.Thesis. Insitut National des Telecommunications, Evry, France.

[10] Cavalli, A.R., Prokopenko, S., Yevtushenko, N. (2000), Fault detection power of a wide test suite for a system of communicating FSMs. Proceedings of 13th IWTCS, pp: 35-56.

[11] Lee, D., Sabnani, K.K., Kristol, D.M., and Paul, S. (1996), Conformance testing of protocols specified as communicating finite state machines - a guided random walk based approach. IEEE Transactions on Communications, 44(5): 631-640.

[12] Gill, A., Introduction to automata theory. Mc Graww-Hill, NY, 1962.

[13] Petrenko, A., Yevtushenko, N., v. Bochmann, G., and Dssouli, R. (1996), Testing in context: Framework and test derivation. Computer Communications Journal, Special issue on protocol engineering, pp: 1236-1249.

[14] Information technology (1991), Open systems interaction. Conformance testing methodology and framework. International standard IS-9646.

[15] Petrenko, A., Yevtushenko, N., and Dssouli, R. (1994), FSM based strategies for testing communicating FSMs. Proceedings of 7th IWTCS.

[16] C. Besse, A. Cavalli, and D. Lee, Optimization Techniques and Automatic Test Generation for TCP Protocols. IEEE Automated Software Engineering, Conference ASE'99, Cocoa Beach, Florida (USA), 12-15 October 1999.

[17] Yevtushenko, N., Cavalli, A.R., and Anido, R. (1999), Test suite minimization for embedded nondeterministic finite state machines. Proceedings of 12th IWTCS, pp: $237-$ 250.

[18] I. Koufareva, Using non-deterministic FSMs for test suite derivation. Ph.D.Thesis, Tomsk State University, Russia, 2000 (In Russian).

[19] Chow, T.S. (1978), Test software design modeled by finite state machines. IEEE Transactions on Software Engineering, 4(3): 178-187.

[20] Fujiwara, S., v.Bochmann,G., Khendek, F., Amalou, M., and Ghendamsi, A. (1991), Test selection based on finite state models. IEEE Transactions on Software Engineering, 17(6): 591-603.

[21] C.Besse, A. Cavalli, M. Kim, F. Zaidi, Two methods for interoperability tests generation. An application to the TCP/IP protocol. Research report N01002-LOR, INT, June 2001.

[22] C. Viho, S. Barbin and L. Tanguy, Towards a formal framework for interoperability testing. Proceedings of Joint Inter. Conf. FORTE2001, pp: 53-68.

[23] J.Zhu, S.T.Vuong, and S.T.Chanson, Evaluation of test coverage for embedded system testing. Proceedings of 11th IWTCS, pp: 111-126. 